\title{
Gaussian Mixture Density based Analytical Model of Noise Induced Variation in Key Parameter of Electronically Tunable Device
}

\author{
Rawid Banchuin
}

\begin{abstract}
In this research, the Gaussian mixture density based analytical model of variation in key parameter of electronically tunable device has been originally proposed. The proposed model is applicable to any electronically tunable device with its tuning variable has been affected by any kind of noise with arbitrary parameters. It has been found from the verification by using different electronically tunable device based empirical distributions and the Kolmogorov-Smirnov tests that this novel model is very accurate. So, it has been found to be a convenient mathematical tool for the analysis and design of various electronically tunable device based circuits.
\end{abstract}

Keywords-Electronically tunable device, Gaussian mixture density, Multiple order distribution, Non-Gaussian noise.

\section{INTRODUCTION}

Electronically tunable device for example, operational transconductance amplifier (OTA) [1, 2], current conveyor $[3,4]$, active resistor $[5,6]$ and the recently proposed voltage differencing differential difference amplifier (VDDDA) [7] etc., has been found to be applicable in many electronic circuits, for example filters [8]-[12], oscillators $[13,14]$, phase shifter [15] and full wave rectifier [16]. The key parameter of these devices for example the transconductance, $g_{m}$ of the OTA etc., has been found to be a function of the tuning variable of the device which can be either voltage or current. Theoretically, perfect tuning which zero variation in key parameter can be obtained is possible. This is because the tuning variable can be assumed to be noise free. Practically, such tuning variable is corrupted by noise. Hence, the perfect tuning mentioned above can never be achieved as noise induced variation in key parameter always exist. This variation is a random process as well as noise and its occurrence is a crucial issue in the analysis and design of any electronically tunable device involved circuit. So, the modeling of the behavior of such variation has been found to be beneficial.

According to this motivation, a novel analytical model of variation in key parameter of electronically tunable device induced by noise in the tuning variable has been proposed in this research. Its derivation has taken multiple time instances into account for completeness without regarding to any specific device and type of tuning variable. This model is applicable to any electronically tunable device either tuned by voltage or current even though such device

Manuscript received February 12, 2015, revised March 26, 2015.

Rawid Banchuin is with the Department of Computer Engineering, Siam University, Bangkok 10160, Thailand (e-mail: rawid.ban@siam.edu). 10.11601/ijates.v4i1.111 may contain specific component e.g. Floating Gate MOSFET etc. It is also applicable to device affected by any kind of noise with arbitrary parameters even though such noise may be non-Gaussian [17]-[19], for example, randomtelegraph-signal noise $[18,19]$ etc. This is because this model has been derived based on the renowned Gaussian mixture density [20] which has been used for analytical modeling of many non-Gaussian distributions [21]-[24]. Furthermore, the verification by using different electronically tunable device based empirical distributions as the references and the Kolmogorov-Smirnov tests states that this originally proposed model has been found to be very accurate. Hence, it has been found to be a convenient mathematical tool for the analysis and design of various electronically tunable device involved circuits.

\section{II.DERIVATION OF The Proposed MODEL}

In this section, derivation of the proposed model will be presented. Obviously, any electronically tunable device is controlled by the tuning variable, $u$ and generate the resulting key parameter $x(u)$ where $u$ can be either voltage or current and $x(u)$ can be any parameter of the device under consideration for example, $x(u)=g_{m}$ if such device is a transconductor/OTA etc.

As $u$ is corrupted by noise denoted by $n(t)$ in practice, noise induced variation in key parameter, $\Delta x(t)$, unfortunately arises and can be defined as

$$
\Delta x(t)=x(u+n(t))-x(u)
$$

Since $u$ is extremely small due to the currently renowned low voltage low power (LVLP) design trend, $\Delta x(t)$ can be given by

$$
\Delta x(t)=x^{\prime}(u) n(t)
$$

where $x^{\prime}(u)$ stands for the derivative of $x(u)$ with respected to $u$. It should be mentioned here that $\Delta x(t)$ is a random process as well as $n(t)$.

In order to analytically model the behavior of $\Delta x(t)$ by taking multiple time instances into account, its multiple order distribution i.e. $N^{\text {th }}$ order distribution where $N \geq 1$ has been found to be convenient. So, it is chosen as the proposed model. For its derivation, the $N^{\text {th }}$ order distribution of $n(t)$ must be firstly determined. Since $n(t)$ can be either voltage or current as well as $u$ which means that $n(t)$ is an electrical quantity, $n(t)$ is stationary $[18,25]$ and has been 
assumed to be independent, identically distributed. As a result, the $N^{\text {th }}$ order distribution of $n(t)$ can be analytically given as follows

$$
\begin{aligned}
& f_{n}\left(n\left(t_{0}\right), t_{0} ; n\left(t_{0}+\tau\right), t_{0}+\tau, . . ; n\left(t_{0}+(N-1) \tau, t_{0}+(N-1) \tau\right)\right) \\
& =\prod_{k=0}^{N-1}\left[f_{n}\left(n\left(t_{0}+k \tau\right), t_{0}+k \tau\right)\right],
\end{aligned}
$$

where $t_{0}$ and $f_{n}\left(n\left(t_{0}+k \tau\right), t_{0}+k \tau\right)$ stand for the initial time instant and the distribution of $n\left(t_{0}+k \tau\right)$ which denotes noise value at any $k^{\text {th }}$ time instant. It can be seen that totally $N$ time instances i.e. $t_{0}, t_{0}+\tau, \ldots t_{0}+(N-1) \tau$, have been now taken into consideration. These time instances are equally distributed on time axis because any pair of $t_{0}+k \tau$ and $t_{0}+(k+1) \tau$ are obviously separated apart by equal time interval given by $\tau$.

Since $n(t)$ can actually be non-Gaussian with arbitrary parameters, it is appropriate to analytically model the distribution of $n\left(t_{0}+k \tau\right)$ by a Gaussian mixture density as this density can model the distribution of any non-Gaussian random variation. So, $f_{n}\left(n\left(t_{0}+k \tau\right), t_{0}+k \tau\right)$ can be given by

$$
f_{n}\left(n\left(t_{0}+k \tau\right), t_{0}+k \tau\right)=\sum_{i=1}^{M}\left[P_{i} g_{n i}\left(n\left(t_{0}+k \tau\right), t_{0}+k \tau\right)\right]
$$

where $g_{n i}\left(n\left(t_{0}+k \tau\right), t_{0}+k \tau\right)$ denotes the $i^{\text {th }}$ Gaussian density component of $n\left(t_{0}+k \tau\right)$ which is a Gaussian density function with $\mu_{n i}$ and $\sigma_{n i}$ as mean and standard deviation, $M$ denotes the size of $f_{n}\left(n\left(t_{0}+k \tau\right), t_{0}+k \tau\right)$ which can be defined as number of components of $f_{n}\left(n\left(t_{0}+k \tau\right), t_{0}+k \tau\right)$ and $P_{i}$ stands for the weight of $g_{n i}\left(n\left(t_{0}+k \tau\right), t_{0}+k \tau\right)$.

With (2)-(4), the above definition of $g_{n i}\left(n\left(t_{0}+k \tau\right), t_{0}+k \tau\right)$ and the principle of random variable transformation which states that if $Y$ is function of $X$, the distributions of $X$ and $Y$ are related by

$$
\int_{-\infty}^{y} g_{Y}(v)|d v|=\int_{-\infty}^{x(y)} f_{X}(u)|d u|
$$

the proposed model i.e. $N^{\text {th }}$ order distribution of $\Delta x(t)$ can be derived as follows

$$
\begin{aligned}
& f_{\Delta x}\left(\Delta x\left(t_{0}\right), t_{0} ; \Delta x\left(t_{0}+\tau\right), t_{0}+\tau, . . ; \Delta x\left(t_{0}+(N-1) \tau, t_{0}+(N-1) \tau\right)\right) \\
& =\prod_{k=0}^{N-1}\left[f_{\Delta x}\left(\Delta x\left(t_{0}+k \tau\right), t_{0}+k \tau\right)\right],
\end{aligned}
$$

where $f_{\Delta x}\left(\Delta x\left(t_{0}+k \tau\right), t_{0}+k \tau\right)$ denotes the distribution of $\Delta x\left(t_{0}+k \tau\right)$ which is the value of $\Delta x(t)$ at any $k^{\text {th }}$ time instant. Of course, $f_{\Delta x}\left(\Delta x\left(t_{0}+k \tau\right), t_{0}+k \tau\right)$ can be given by

$$
\begin{aligned}
& f_{\Delta x}\left(\Delta x\left(t_{0}+k \tau\right), t_{0}+k \tau\right) \\
& =\sum_{i=1}^{M}\left[\frac{P_{i}\left[\left|x^{\prime}(u)\right|\right]^{-1}}{\sqrt{2 \pi} \sigma_{n i}} \exp \left[-\frac{\left[\Delta x\left(t_{0}+k \tau\right)-x^{\prime}(u) \mu_{n i}\right]^{2}}{2\left[x^{\prime}(u)\right]^{2} \sigma_{n i}^{2}}\right]\right] .
\end{aligned}
$$

Since $n(t)$ is stationary which means that its parameters such as $P_{i}, \mu_{n i}$ and $\sigma_{n i}$ etc., are time invariant, and $x^{\prime}(u)$ is time independent, $\Delta x\left(t_{0}+k \tau\right)$ is identically distributed for any $k \in\{0,1,2, \ldots, N-1\}$. This means that $\Delta x(t)$ is independent, identically distributed and also stationary.

At this point, the proposed model has been already derived. It is applicable to the device affected by noise of any kind with arbitrary parameters because the Gaussian mixture density has been adopted as the basis. It is also applicable to the device affected by Gaussian noise by simply letting $M=1$ and $P_{i}=1$. Moreover, it can be applied to any electronically tunable device with any kind of tuning variable as $u$ can be either voltage or current and $x(u)$ can be any parameter as mentioned above. This point will be illustrated in the subsequent section which the applications of this model to different electronically tunable devices will be demonstrated. Finally, $x^{\prime}(u)$ can be conveniently determined in a computer aided manner by using various software packages for example, Mathematica, Mathcad and MATLAB etc.

\section{APPLICATIONS OF THE MODEL TO DIFFERENT DEVICES}

Firstly, the application of the model to the voltage controlled current source (VCCS) will be illustrated where a VCCS proposed in [26] will be considered. For this VCCS, the tuning variable is the controlling voltage, $V_{c}=V_{a}-V_{b}$ and the key parameter is the transconductance, $G_{m}\left(V_{c}\right)$. By applying the model and let $u=V_{c}, x(u)=G_{m}\left(V_{c}\right)$ and $\Delta x\left(t_{0}+k \tau\right)=\Delta G_{m}\left(t_{0}+k \tau\right)$ where $\Delta G_{m}\left(t_{0}+k \tau\right)$ denotes the value at any $k^{\text {th }}$ time instant of $\Delta G_{m}(t)$ which stands for noise induced variation in transconductance of this VCCS, the $N^{\text {th }}$ order distribution of $\Delta G_{m}(t)$ can be analytically given as

$$
\begin{aligned}
& f_{\Delta G_{m}}\left(\Delta G_{m}\left(t_{0}\right), t_{0} ; \Delta G_{m}\left(t_{0}+\tau\right), t_{0}+\tau, . . ; \Delta G_{m}\left(t_{0}+(N-1) \tau, t_{0}+(N-1) \tau\right)\right) \\
& =\prod_{k=0}^{N-1}\left[\sum_{i=1}^{M}\left[\frac{P_{i}\left[G_{m}^{\prime}\left(V_{c}\right)\right]^{-1}}{\sqrt{2 \pi} \sigma_{n, i}} \exp \left[-\frac{\left[\Delta G_{m}\left(t_{0}+k \tau\right)-G_{m}^{\prime}\left(V_{c}\right) \mu_{n i}\right]^{2}}{2\left[G_{m}^{\prime}\left(V_{c}\right)\right]^{2} \sigma_{n i}^{2}}\right]\right] .\right.
\end{aligned}
$$

Secondly the application of the proposed model to the active resistor will be shown. Here, the active resistor realized by using a special kind of MOSFET namely Floating Gate MOSFET (FGMOSFET) proposed in [27] will be considered. For this active resistor, the tuning variable and the key parameter have been found to be the controlling voltage, $V_{c}$ and the equivalent resistance, $R_{E C H}\left(V_{c}\right)$ respectively. By applying the model and let $u=V_{c}$, $x(u)=R_{E C H}\left(V_{c}\right)$ and $\Delta x\left(t_{0}+k \tau\right)=\Delta R_{E C H}\left(t_{0}+k \tau\right)$ where $\Delta R_{E C H}\left(t_{0}+k \tau\right)$ denotes the value at any $\mathrm{k}^{\text {th }}$ time instant of $\Delta R_{E C H}(t)$ which stands for the noise induced variation in the equivalent resistance of this active resistor, the $N^{\text {th }}$ order distribution of $\Delta R_{E C H}(t)$ can be analytically found as follows

$$
\begin{aligned}
& f_{\Delta R_{E C H}}\left(\Delta R_{E C H}\left(t_{0}\right), t_{0} ; \Delta R_{E C H}\left(t_{0}+\tau\right), t_{0}+\tau, . . ; \Delta R_{E C H}\left(t_{0}+(N-1) \tau, t_{0}+(N-1) \tau\right)\right) \\
& =\prod_{k=0}^{N-1}\left[\sum_{i=1}^{M}\left[\frac{P_{i}\left[\left|R_{E C H}^{\prime}\left(V_{c}\right)\right|\right]^{-1}}{\sqrt{2 \pi} \sigma_{n, i}} \exp \left[-\frac{\left[\Delta x\left(t_{0}+k \tau\right)-R_{E C H}^{\prime}\left(V_{c}\right) \mu_{n i}\right]^{2}}{2\left[R_{E C H}^{\prime}\left(V_{c}\right)\right]^{2} \sigma_{n i}^{2}}\right]\right]\right]
\end{aligned}
$$

Next, the application of the model to the current conveyor will be illustrated where the $2^{\text {nd }}$ generation current controlled current conveyor (CCCII) proposed in [3] has been chosen for consideration. For this current conveyor, the tuning variable is the biasing current, $I_{O}$ and the key parameter is the parasitic resistance at port $\mathrm{X}$ of the CCCII, 
$R_{X}\left(I_{o}\right)$. Similarly to the previous cases, the $N^{\text {th }}$ order distribution of noise induced variation in parasitic resistance, $\Delta R_{X}(t)$ can be determined by applying the model. In order to do so, we must let $u=I_{o}, x(u)=R_{X}\left(I_{o}\right)$ and $\Delta x\left(t_{0}+k \tau\right)=\Delta R_{X}\left(t_{0}+k \tau\right)$ where $\Delta R_{X}\left(t_{0}+k \tau\right)$ denotes the value at any $k^{\text {th }}$ time instant of $\Delta R_{X}(t)$. As a result, the $N^{\text {th }}$ order distribution of $\Delta R_{X}(t)$ can be analytically given by

$$
\begin{aligned}
& f_{\Delta R_{X}}\left(\Delta R_{X}\left(t_{0}\right), t_{0} ; \Delta R_{X}\left(t_{0}+\tau\right), t_{0}+\tau, . . ; \Delta R_{X}\left(t_{0}+(N-1) \tau, t_{0}+(N-1) \tau\right)\right) \\
& =\prod_{k=0}^{N-1}\left[\sum_{i=1}^{M}\left[\frac{P_{i}\left[\left[R_{X}^{\prime}\left(I_{o}\right)\right]\right]^{-1}}{\sqrt{2 \pi} \sigma_{n, i}} \exp \left[-\frac{\left[\Delta x\left(t_{0}+k \tau\right)-R_{X}^{\prime}\left(I_{o}\right) \mu_{n i}\right]^{2}}{2\left[R_{X}^{\prime}\left(I_{o}\right)\right]^{2} \sigma_{n i}^{2}}\right]\right]\right]
\end{aligned}
$$

Finally, the application of this model to the VDDDA will be shown where the VDDDA proposed in [7] will be considered. In this case, the tuning variable is the control current, $I_{B}$ and the key parameter is the transconductance, $g_{m}\left(I_{B}\right)$. Similarly to the previous cases, the $N^{\text {th }}$ order distribution of noise induced variation in the transconductance, $\Delta g_{m}(t)$ can be analytically determined by applying the model. In order to do so, we must let $u=I_{B}$, $x(u)=g_{m}\left(I_{B}\right)$ and $\Delta x\left(t_{0}+k \tau\right)=\Delta g_{m}\left(t_{0}+k \tau\right)$ where $\Delta g_{m}\left(t_{0}+k \tau\right)$ denotes the value at any $k^{\text {th }}$ time instant of $\Delta g_{m}(t)$. As a result, the $N^{\text {th }}$ order distribution of $\Delta g_{m}(t)$ can be analytically given as follows

$$
\begin{aligned}
& f_{\Delta g_{m}}\left(\Delta g_{m}\left(t_{0}\right), t_{0} ; \Delta g_{m}\left(t_{0}+\tau\right), t_{0}+\tau, . . ; \Delta g_{m}\left(t_{0}+(N-1) \tau, t_{0}+(N-1) \tau\right)\right) \\
& =\prod_{k=0}^{N-1}\left[\sum_{i=1}^{M}\left[\frac{\left.P_{i}\left[g_{m}^{\prime}\left(I_{B}\right)\right]\right]^{-1}}{\sqrt{2 \pi} \sigma_{n, i}} \exp \left[-\frac{\left[\Delta x\left(t_{0}+k \tau\right)-g_{m}^{\prime}\left(I_{B}\right) \mu_{n i}\right]^{2}}{2\left[g_{m}^{\prime}\left(I_{B}\right)\right]^{2} \sigma_{n i}^{2}}\right]\right]\right] .
\end{aligned}
$$

At this point, it can be seen that the distributions of the variations in key parameters of different electronically tunable devices i.e. VCCS, active resistor current conveyor and VDDDA can be analytically obtained in the similar manner by applying the proposed model. Apart from these devices, this model can be applied to other electronically tunable devices as well for example, OPAMP, active inductor [13], Operational Transresistance Amplifier (OTRA) [14, 28], Current Difference Transconductance Amplifier (CDTA) [16], Current Differencing Buffered Amplifier (CDBA) [29], Voltage Differencing Transconductance Amplifier (VDTA) [30], Voltage Differencing Buffered Amplifier (VDBA) [31], Voltage Differencing Current Conveyor (VDCC) [32] and active transformer [33] etc. This is because it has been derived regardless to any specific device. Furthermore it is also applicable to those devices based on any special component apart from the FGMOSFET such as FinFET [34] and Carbon nanotube FET (CNTFET) [35] etc., since it has been derived regardless to any specific component.

\section{The Model VerificATION}

In this section, the verification of the proposed model will be addressed. It should be mentioned here that the $0.25 \mu \mathrm{m}$ CMOS process technology of TSMC has been adopted as the verification basis. Since $\Delta x(t)$ is stationary which means that its behavior is time independent, it is sufficient to perform the verification at an arbitrary instant since the obtained results are always valid. Such verification has been performed based on the VCCS, active resistor, current conveyor (CCCII) and VDDDA proposed in [26], [27], [3] and [7] respectively. It has been assumed the tuning variable of the active resistor is corrupted by the impulse noise (random telegraph noise) $[18,19]$ where those of the VCCS and the current conveyor are respectively corrupted by the student-t noise and the Rayleigh noise which can occur since noise can be non-Gaussian [17]-[19]. On the other hand, the tuning variable of the VDDDA has been assumed to be corrupted by the Gaussian noise in in order to verify the accuracy of the model when applied to the device affected by such noise.

For the verification in the qualitative manner, the analytically determined distributions of $\Delta G_{m}(t), \Delta R_{E C H}(t)$, $\Delta R_{x}(t)$ and $\Delta g_{m}(t)$ obtained by using the proposed model have been comparatively plotted against their corresponding references which are the electronically tunable device based empirical distributions obtained by using the $0.25 \mu \mathrm{m}$ level SPICE BSIM3v3 based Monte-Carlo simulations with parameters provided by MOSIS. On the other hand, the verification in the quantitative manner has been performed via the KS-tests [36, 37] of these analytically obtained distributions. The KS-test has been chosen due to its simpler statistic compared to the others and its capability to fit nonGaussian distributions [37]. In general, the KS-test of any distribution can be performed by comparing its statistic, $K S$ to the critical value, $c$ where it can be stated that the distribution under test fits the empirical distribution at any certainly specified confidence level if and only if $K S \leq c$. Mathematically, KS can be given by

$$
K S=\max _{x}\left\{\left|\int_{-\infty}^{x} f_{X}^{\prime}(u) d u\right|-\left|\int_{-\infty}^{x} f_{X}(u) d u\right|\right\},
$$

where $f_{X}(x)$ and $f_{X}^{\prime}(x)$ denote the distribution under test and the empirical distribution respectively.

Since confidence level of the test is chosen to be $99 \%, c$ can be given by [36]

$$
c=\frac{1.63}{\sqrt{n}}
$$

where $n$ denotes the number of runs of each Monte-Carlo simulation. It should be mentioned here that $c=0.02976$ because $n=3000$. At this point, the model verification based on VCCS, active resistor, current conveyor and VDDDA will be subsequently presented.

\section{A. The verification based on the VCCS affected by the} student-t noise

In this subsection, the verification based on the VCCS with $V_{c}$ corrupted by the student-t noise, will be presented. Since it is assumed that the equivalent mean and equivalent standard deviation of noise are $0 \mathrm{~V}$ and $1.4239 \mathrm{nV}$ respectively, its distribution can be analytically modeled by using a Gaussian mixture density. By letting $M=5$ and applying the maximum likelihood parameter estimation, it has been found that $P_{1}=0.31, P_{2}=0.005, P_{3}=0.68, P_{4}=P_{5}$ 
$=0.0025, \mu_{n 1}=\mu_{n 2}=\mu_{n 3}=\mu_{n 4}=\mu_{n 5}=0 \mathrm{~V}, \sigma_{n 1}=\sqrt{2} \mathrm{nV}$, $\sigma_{n 2}=\sqrt{20} \mathrm{nV}, \sigma_{n 3}=\sqrt{1.5} \mathrm{nV}, \sigma_{n 4}=\sqrt{15} \mathrm{nV}$ and $\sigma_{n 5}=\sqrt{10}$ $\mathrm{nV}$. For the verification in the qualitative sense, the analytically determined distribution of $\Delta G_{m}(t)$ has been comparatively plotted against its reference which is the VCCS based empirical distribution, as shown in Fig. 1 where the horizontal axis shows that the unit of $\Delta G_{m}(t)$ is the percentage of the nominal $G_{m}\left(V_{c}\right)$ which $V_{c}$ is noise free. From Fig.1, a strong agreement between the analytically determined distribution and its reference can be observed.

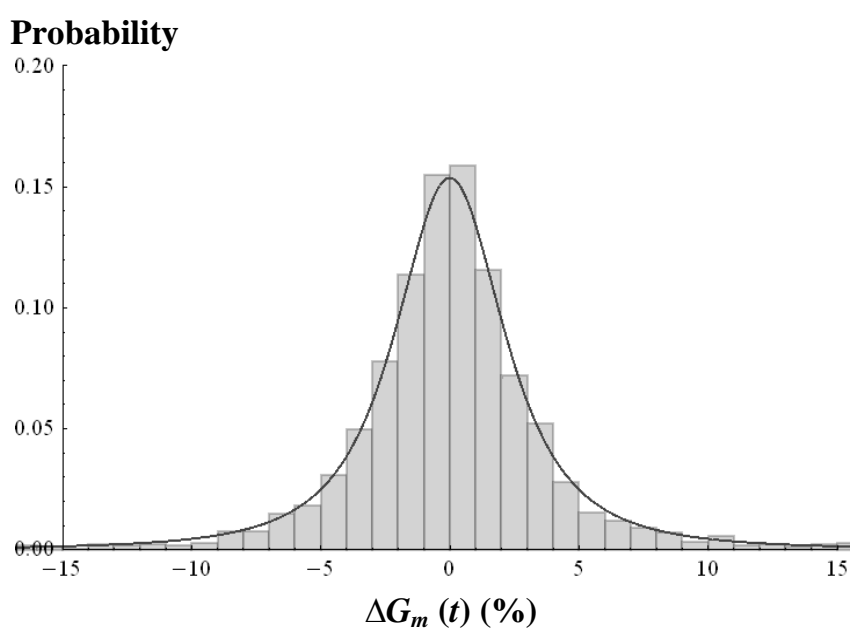

Fig.1. Analytically determined distribution of $\Delta G_{m}(t)$ (line) v.s. The VCCS based empirical distrubution (histogram)

For the verification in the quantitative manner, the KStest of the analytically determined distribution of $\Delta G_{m}(t)$ has been performed. In this case, such distribution serves as $f_{X}(x)$ where the VCCS based empirical distribution serves as $f_{X}^{\prime}(x)$. By using (12), it has been found that $K S=$ 0.00195 which satisfies $K S \leq c$ as $c=0.02976$. This means that the analytically determined distribution fits its reference with $99 \%$ confidence. Since the results of the verification in both qualitative and quantitative manners have been found to be very pleasant, the accuracy of the proposed model has been now verified.

Furthermore, the analytically determined distribution of $\Delta G_{m}(t)$ shows that $\Delta G_{m}(t)$ has a student-t distribution with zero equivalent mean as the VCCS based empirical distribution does. $\Delta G_{m}(t)$ has zero equivalent mean because $V_{c}$ is corrupted by noise with zero equivalent mean. The analytically determined distribution and the empirical distribution give the equivalent standard deviations of $7.27 \%$ and $7.06 \%$ respectively. It can be seen that these standard deviations are very close to each other. This also verifies the accuracy of the proposed model.

$B$. The verification based on the active resistor affected by the impulse noise

In this subsection, the verification based on the active resistor with $V_{c}$ corrupted by the impulse noise will be presented. Here, it has been assumed that such impulse noise has two levels.

Since the distribution of any two level impulse random signal, $s(t)$ which the value of each level is deterministic, can be given by $h(s(t), t)=\left\{\begin{array}{l}P \\ 1-P \\ 0\end{array}\right.$

$$
\begin{aligned}
& ; s(t)=S_{1} \\
& ; s(t)=S_{2} \\
& ;\left(s(t) \neq S_{1}\right) \wedge\left(s(t) \neq S_{2}\right)
\end{aligned}
$$

where $S_{1}$ and $S_{2}$ are certain possible deterministic values of $s(t)$, the distribution of two level impulse noise can be analytically modeled by using Gaussian mixture density with $M=2, P_{1}=P$ and $P_{2}=1-P$. Since the equivalent mean and equivalent standard deviation of such noise are assumed to be $80 \mathrm{nV}$ and $89.445 \mathrm{nV}$ respectively, it has been found that $P_{1}=0.5, P_{2}=0.5, \mu_{n 1}=40 \mathrm{nV}, \mu_{n 2}=120 \mathrm{nV}, \sigma_{n 1}=$ $\sqrt{0.6} \mathrm{nV}$ and $\sigma_{\mathrm{n} 2}=\sqrt{0.4} \mathrm{nV}$ by using the maximum likelihood parameter estimation.

As the verification in the qualitative manner, the analytically determined distribution of $\Delta R_{E C H}(t)$ has been comparatively plotted against its reference i.e. the active resistor based empirical distribution as shown in Fig.2 where the horizontal axis shows that the unit of $\Delta R_{E C H}(t)$ is the percentage of the nominal $R_{E C H}\left(V_{c}\right)$ which $V_{c}$ is noise free. From Fig.2, a strong agreement between the analytical distribution and its reference can be observed.

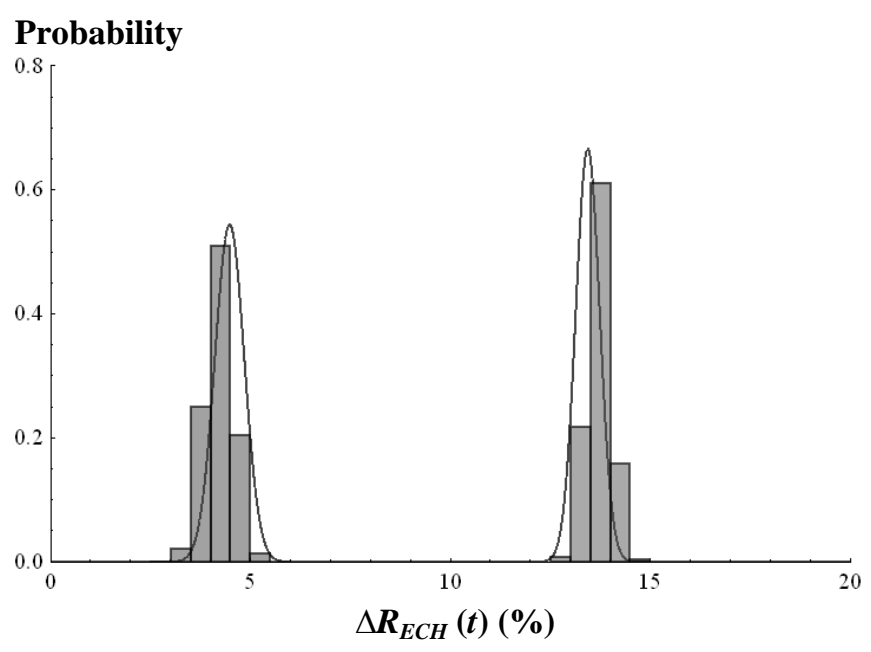

Fig.2. Analytically determined distribution of $\Delta R_{E C H}(t)$ (line) v.s. The active resistor based empirical distribution (histogram)

As the verification in the quantitative sense, the KS-test of the analytically determined distribution of $\Delta R_{E C H}(t)$ has been performed where such distribution serves as $f_{X}(x)$ and the active resistor based empirical distribution serves as $f_{X}^{\prime}(x)$ in this case. By using (12), it has been found that $K S=0.018$ which also satisfies $K S \leq c$ as $c=0.02976$. This means that the analytically determined distribution fits its reference with 99\% confidence. As the results of the verification in both qualitative and quantitative manners have been found to be very pleasant, the proposed model has been found to be accurate.

Moreover, both analytically determined distribution of $\Delta R_{E C H}(t)$ and active resistor based empirical distribution show that $\Delta R_{E C H}(t)$ has a Gaussian mixture distribution with two levels. The analytically determined distribution gives the equivalent mean and standard deviation of $8.96 \%$ and $10.02 \%$ respectively. On the other hand, the equivalent mean and standard deviation obtained from the empirical distribution can be respectively given by $8.42 \%$ and $9.42 \%$. 
It can be seen that these sets of parameters are very close to each other. This also verifies the accuracy of the model.

C. The verification based on the current conveyor affected by the Rayleigh noise

In this subsection, the verification based on the current conveyor i.e. CCCII with $I_{o}$ corrupted by the Rayleigh noise will be presented. It has been assumed that the equivalent mean and equivalent standard deviation of noise are given by $60.553 \mathrm{nA}$ and $31.623 \mathrm{nA}$ respectively. For the verification in the qualitative manner, the analytically determined distribution of $\Delta R_{X}(t)$ has been comparatively plotted against its reference i.e. the current conveyor based empirical distribution as shown in Fig.3 where the unit of $\Delta R_{X}(t)$ is the percentage of the nominal $R_{X}\left(I_{o}\right)$ which $I_{o}$ is noise free. From Fig.3, a strong agreement between the analytically determined distribution and its reference can also be seen.

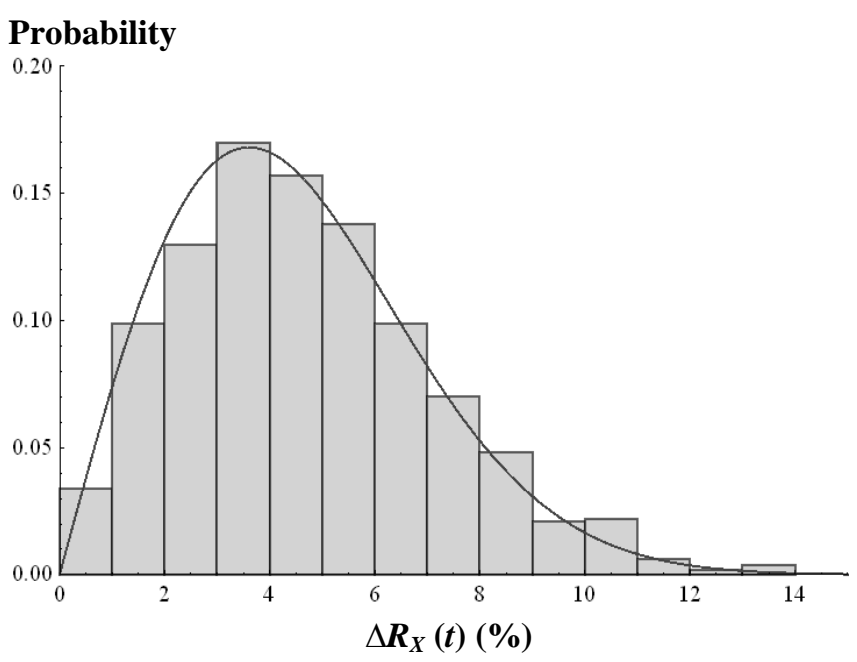

Fig.3. Analytically determined distribution of $\Delta R_{X}(t)$ (line) v.s. The current conveyor based empirical distribution (histogram)

As the verification in the quantitative sense, the KS-test of the analytically determined distribution of $\Delta R_{X}(t)$ has been performed. Now, such distribution and the current conveyor based empirical distribution serves as $f_{X}(x)$ and $f_{X}^{\prime}(x)$ respectively. By using (12), it has been found that $K S=0.022$ which also satisfies $K S \leq c$ as $c=0.02976$. This means that the analytically determined distribution fits its reference with $99 \%$ confidence. At this point, the accuracy of the proposed model has been now verified according to the soundness of the results of verification in both senses mentioned above.

Moreover, both analytically determined distribution of $\Delta R_{X}(t)$ and active resistor based empirical distribution show that $\Delta R_{X}(t)$ has a Rayleigh distribution. The analytically determined distribution gives the equivalent mean and standard deviation of $4.523 \%$ and $2.364 \%$ respectively. On the other hand, the equivalent mean and standard deviation obtained from the empirical distribution can be respectively given by $4.72 \%$ and $2.465 \%$. It can be seen that these sets of statistical parameters are very close to each other. This alternatively verifies the accuracy of the proposed model.

\section{D.The verification based on the VDDDA affected by the} Gaussian noise

Now, the verification based on the VDDDA with $I_{B}$ corrupted by the Gaussian noise will be presented. It has been assumed that mean and standard deviation of such noise are $0 \mathrm{~V}$ and $1.1559 \mathrm{nV}$ respectively. For the verification in the qualitative sense, the analytically determined distribution of $\Delta g_{m}(t)$ has been comparatively plotted against its reference which is the VDDDA based empirical distribution, as shown in Fig. 4 where the unit of $\Delta g_{m}(t)$ is the percentage of the nominal $g_{m}\left(I_{B}\right)$ which $I_{B}$ is noise free. From Fig.4, a strong agreement between the analytical distribution and its reference can be observed.

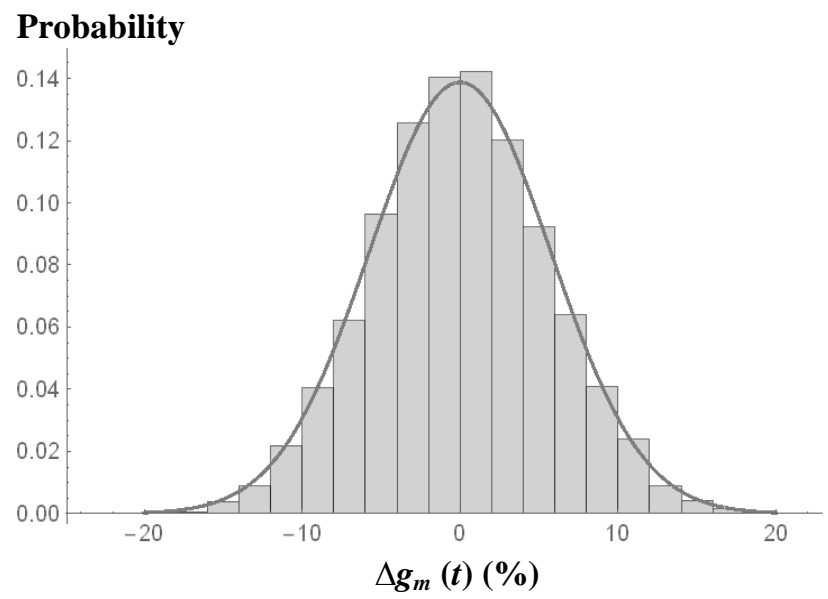

Fig.4. Analytically determined distribution of $\Delta g_{m}(t)$ (line) v.s. The VDDDA based empirical distribution (histogram)

For the verification in a quantitative manner, the KS-test of the analytical distribution of $\Delta g_{m}(t)$ has been performed where such distribution serves as $f_{X}(x)$ and the VDDDA based empirical reference serves as $f_{X}^{\prime}(x)$. By using (12), it has been found that $K S=0.00225$ which satisfies $K S \leq c$ as $c=0.02976$. This means that the analytically determined distribution fits its reference with $99 \%$ confidence. So, the proposed model has been found to be very accurate according to the soundness of the results of verification in both manners.

Furthermore, the analytically determined distribution of $\Delta g_{m}(t)$ states that $\Delta g_{m}(t)$ has a zero mean Gaussian distribution as the VDDDA based empirical distribution does. Such distribution of $\Delta g_{m}(t)$ is obtained because $I_{B}$ is corrupted by a zero mean Gaussian noise. The analytically determined distribution and its reference give the standard deviations of $5.75 \%$ and $5.53 \%$ respectively which are very close to each other. This also verifies the accuracy of the proposed model.

\section{V.CONCLUSION}

The Gaussian mixture density based analytical model of the variation in key parameter of any electronically tunable device induced by noise in tuning voltage/current which can be any kind with arbitrary parameters, has been originally derived in this research. Its applications to different electronically tunable devices have been demonstrated. The verification performed by using different electronically 
tunable device based empirical distributions as the references and the 99\% confidence level KS-tests states that this model is very accurate. Hence, it has been found to be beneficial to the analysis and design of various circuits involving electronically tunable devices.

\section{ACKNOWLEDGMENT}

The author would like to acknowledge Mahidol University, Thailand, for the online database service.

\section{REFERENCES}

[1] E Sanchez-Sinencio and J. Silva-Martinez, "CMOS transconductance amplifiers, architectures and active filters: a tutorial," IEE Proc. Circuits, Devices and Systems, vol.147, pp. 3-12, 2000.

[2] T.-Y. Lo and C.-C. Hung, "A 1-V 50-MHz pseudodifferential OTA with compensation of the mobility reduction,” IEEE Trans. Circuits and Systems II: Express Briefs, vol. 54, pp. 1047-1051, 2007

[3] R. Chaisricharoen, B. Chipipop and B. Sirinaovakul, "CMOS CCCII: structures, characteristics and considerations," AEÜ - Int. J. Electronics and Communications, vol.64, pp. 540-557, 2010.

[4] T. Kurashina, S. Ogawa and K. Watanabe, "A CMOS rail-to-rail current conveyor," IEICE Trans. Fundamental Electronics, Communications and Computer Sciences, vol.E85-A, pp. 2894-2900, 2002

[5] S.A. Mahmoud, "Low voltage CMOS floating resistor," in Proc. 2004 Int. Conf. Electrical, Electronic and Computer Engineering, pp.453456.

[6] C. Copa, "Linear active resistor based on CMOS square-root circuits for VLSI applications," in Proc. EUROCON'2005 Int. Conf. Computer as a Tool, pp.894-897.

[7] N. Herencsar, R. Sotner, B. Metin, J. Koton and K. Vrba, "VDDDANew voltage differencing device for analog signal processing," in Proc. ELECO'2013 $8^{\text {th }}$ Int. Conf. Electrical and Electronics Engineering, pp.17-20.

[8] T. Kurashina, S. Ogawa and K. Watanabe, “A CMOS rail-to-rail current conveyor and its applications to current-mode filters,” IEICE Trans. Fundamental Electronics, Communications and Computer Sciences, vol.E86-A, pp.1445-1450, 2003

[9] X. Zhang and E. I. El-Masry, " A novel CMOS OTA based on bodydriven MOSFETs and its applications in OTA-C filters,” IEEE Trans. Circuits and Systems I: Regular Papers, vol. 54, pp.1204-1212, 2007

[10] R.K. Ranjan, S.P. Yalla, S. Sorya, and S.K. Paul, “Active comb filter using operational transconductance amplifier,” Active and Passive Electronic Components, vol.2014, pp.1-6, 2014.

[11] N. Pandey, D. Nand, and Z. Khan, "Single-input four-output current mode filter using operational floating current conveyor," Active and Passive Electronic Components, vol.2013, pp.1-8, 2013.

[12] J. Koton, N. Herencsar, K. Vrba and B. Metin, "The VDDDA in multifunction filter with mutually independent $Q$ and $\omega_{0}$ control featrure," in Proc. ELECO'2013 $8^{\text {th }}$ Int. Conf. Electrical and Electronics Engineering, pp.53-56.

[13] L. H. Lu, H. H. Hsieh, and Y. T. Liao, “A wide tuning-range CMOS VCO with differential tunable active inductor, " IEEE Trans. Microwave Theory and Techniques, vol. 54, pp. 3462-3468, 2006.

[14] H.-C. Chien, "New realizations of single OTRA-based sinusoidal oscillators," Active and Passive Electronic Components, vol.2014, pp.1-12, 2014.

[15] Y. Zheng and C. E. Saavedra, "An ultra-compact CMOS variable phase shifter for $2.4 \mathrm{GHz}$ ISM applications," IEEE Trans. Microwave Theory and Techniques, vol. 56, pp. 1349-1354, 2008

[16] N. Pandey and R. Pandey, "Current mode full-wave rectifier based on a single MZC-CDTA," Active and Passive Electronic Components, vol.2013, pp.1-5, 2013.

[17] M.M. Jevtic, "Noise as a diagnostic and prediction tool in reliability physics,” Microelectronic Reliability, vol. 35, pp. 455-477, 1995

[18] G. Ghibaudo, T. Boutchacha, "Electrical noise and RTS fluctuations in advanced CMOS devices,” Microelectronic Reliability, vol. 42, pp. 573-582, 2002

[19] M. Haartman and M. Ostling, Low Frequency Noise in Advanced MOS Devices. Netherlands: Springer, 2007

[20] J. Behboodian, "On a mixture of normal distributions," Biometrika, vol. 57, pp. 215-217, 1970
[21] Y. Zhao, X. Zhuang, and S. J. Ting, "Gaussian mixture density distribution of non-Gaussian source for autoregressive process,” IEEE Trans. Signal Processing, vol. 43, pp. 894-903, 1995.

[22] R. S. Blum, Y. Zhang, B. M. Sadler, and R. J. Kozick, “On the approximation of correlated non-Gaussian noise PDFs using Gaussian mixture distributions," in Proc. 1999 Conf. Applied Heavy Tailed Distributions on Economic, Engineering and Statistics, pp.1-10.

[23] R. J. Kozick and B. M. Sadler, "Maximum-likelihood array processing in non-Gaussian noise with Gaussian mixtures," IEEE Trans. Signal Pocessing, vol. 48, pp.3520-3535, 2000

[24] A. Bijaoui, "Wavelets, Gaussian mixtures and Wiener filtering," Signal Processing, vol.82, pp 709-712, 2002

[25] S.L. Miller and D.G. Childers, Probability and Random Processes with Applications to Signal Processing and Communications. Burlington, MA: Elsevier, 2004

[26] P. Bertemes-Filho, V. C. Vincence, M. M. Santos and I. X. Zanatta, "Low power current sources for bioimpedance measurements: a comparison between Howland and OTA-based CMOS circuits," J. Electrical Bioimpedance, vol. 3, pp. 66-73, 2012

[27] A. Manolescu and C. Copa, "Low-voltage low-power improved linearity CMOS active resistor circuits,” Analog Integrated Circuits and Signal Processing, vol. 62, pp. 373-387, 2010

[28] J.-J. Chen, H.-W. Tsao and C.-C. Chen, Operational transresistance amplifier using CMOS technology, Electronics Letters, vol. 28, pp. 2087-2088, 1992.

[29] C. Acar and S. Özoğuz, "A versatile building block: current differencing buffered amplifier suitable for analog signal processing filters,” Microelectronics Journal, vol. 30, pp. 157-160, 1999.

[30] A. Yesil, F. Kacar and H. Kuntman, "New simple CMOS realization of voltage differencing transconductance amplifier and its RF filter application,” Radio Engineering, vol. 20, pp. 632-637, 2011.

[31] A. Guney, E. Alaybeyoglu and H. Kuntman, "New CMOS realization of Z copy voltage differencing buffered amplifier and its currentmode filter application,” in Proc. DTIS'2013 $8^{\text {th }}$ Int. Conf. Design \& Technology of Integrated Systems in Nanoscale Era, pp.68-71.

[32] R. Sotner, J. Jerabek, N. Herencsar, T. Dostal and K. Vrba, "Design of Z-copy controlled-gain voltage differencing current conveyor based adjustable functional generator," Microelectronics Journal, vol.46, pp.143-152, 2015

[33] F. Yuan, "CMOS gyrator-C active transformers," IET Circuits Devices and Systems, vol. 1, pp. 494-508, 2007.

[34] Z. Kesilmis, M. Avci and M. Aksoy, "An operational transconductance amplifier with 45nm FINFET technology,” in Proc. ELECO'2007 5th Int. Conf. Electrical and Electronics Engineering, pp.1-4.

[35] S.A. Loan, M. Nizamuddin, F. Bashir, H. Shabir, A.M. Murshid, A.R. Alamoud and S.A. Abbasi, "Design of a novel high gain carbon nanotube based operational transconductance amplifier," in Proc. IMECS'2014 Int. Multi Conf. Engineers and Computer Scientists, pp.1-4.

[36] S.A. Klugman, H.H. Panjer H.H and G.E. Willmot G.E, Loss Models: From Data to Decisions, Hoboken, NJ: John Wiley and Sons, 2008

[37] J. L. Romeu, "Kolmogorov-Simirnov: a goodness of fit test for small samples,” Selected Topics in Assurance Related Technologies, vol. 10, pp.1-6

Rawid Banchuin received the B.Eng. degree in electrical engineering from Mahidol University, Bangkok, Thailand in 2000, the degree of M.Eng. in computer engineering and Ph.D. in electrical and computer engineering from King Mongkut's University of Technology Thonburi, Bangkok, Thailand in 2003 and 2008 respectively.

He was with the Department of Electrical Engineering, Rajamangala Institute of Technology, North-Bangkok Campus since 2003. At the present, he is with the Department of Computer Engineering, Siam University, Bangkok, Thailand.

Asst. Prof. Dr. Rawid Banchuin is a member of council of engineer (Thailand) and has joined the organizing committee of the international conference on ICT and knowledge engineering which is jointly organized by IEEE, since 2012 . 